\title{
Adesivos de lignina Kraft de Eucalyptus spp.
}

\author{
Josué Teixeira ${ }^{1}$, Larissa Carvalho Santos ${ }^{1 *}$, Iris Araújo Silva ${ }^{1}$, Vivian Silva Santos ${ }^{1}$, Eduarda Marcela Algelly \\ Costa Piau ${ }^{1}$, Camila Ferreira Paixão ${ }^{1}$, Sabryna Luiza Rivelli de Oliveira ${ }^{1}$
}

\begin{abstract}
RESUMO: O aumento na produção de celulose na última década acarretou aumento da geração de licor negro, cujo principal componente é a lignina Kraft. A estrutura da lignina Kraft de eucalipto, tipo siringil, é a menos reativa ao formaldeído, devido à presença de grupos metil ligados aos carbonos 3 e 5 das unidades de fenilpropano. Apesar disto, ainda é considerada o substituto mais promissor para os compostos fenólicos não renováveis. Objetivou-se sintetizar adesivos lignina-fenol-formaldeído, caracterizar suas propriedades e avaliar a resistência ao cisalhamento na linha de cola. Para isso, utilizou-se lignina Kraft de eucalipto para sintetizar 4 adesivos (T2, T3, T4 e T5) com substituição de 25, 50,75 e $100 \%$ do fenol, respectivamente. Ademais, foi sintetizado adesivo testemunha, sem substituição do fenol. Foram observadas variações significativas de gel time entre todos os tratamentos, com maior valor em $\mathrm{T} 1$; os valores de $\mathrm{pH}$ diminuiram à medida que a lignina Kraft foi adicionada aos adesivos, com o maior valor obtido para T1; os valores de teor de sólidos também foram estatisticamente diferentes entre si; na viscosidade todos os tratamentos foram estatisticamente diferentes entre-se, com aumento no valor desta propriedade conforme incrementou-se a quantidade de lignina; No teste de cisalhamento, nas condições seca e úmida, T5 e T4 apresentaram os maiores valores de resistência. Concluiu-se que os adesivos T4 e T5 possuem maior potencial para aplicação industrial.
\end{abstract}

Palavras-chave: adesivos fenólicos, lignina Kraft, adesivos de lignina, valorização da lignina Kraft, resistência ao cisalhamento.

\section{Eucalyptus Kraft lignin adhesives}

\begin{abstract}
The increase in cellulose production in the past decade has led to an increase in the generation of black liquor, the main component of which is a Kraft lignin. A structure of eucalyptus Kraft lignin, syringyl type, is the least reactive to formaldehyde, due to the presence of methyl groups linked to carbons 3 and 5 of the phenylpropane units. Despite this, it is still considered the most promising substitute for non-renewable phenolic compounds. The objective was to synthesize lignin-phenol-formaldehyde adhesives, characterize their properties and evaluate the shear strength in the glue line. For this, eucalyptus Kraft lignin is used to synthesize 4 adhesives (T2, T3, T4 and T5) with substitution of $25,50,75$ and $100 \%$ of the phenol, respectively. In addition, substitute adhesive was synthesized without phenol substitution. Variations in gel time were observed between all treatments, with greater value in $\mathrm{T} 1$; $\mathrm{pH}$ values decreased as Kraft lignin was added to the adhesives, with the highest value established for T1; the values of solids content were also statistically different from each other; in viscosity, all treatments were statistically different among themselves, with an increase in the value of this property as the amount of lignin increased; In the shear test, in dry and wet conditions, T5 and T4 give the highest strength values. It was concluded that T4 and T5 adhesives have greater potential for industrial applications.
\end{abstract}

Keywords: phenolic adhesives, Kraft lignin, lignin adhesives, Kraft lignin recovery, shear strength.

\section{INTRODUÇÃO}

A lignina é constituinte da parede celular das plantas, estando concentrada principalmente na lamela média, onde é depositada durante a lignificação dos tecidos (RINALDI et al., 2016). De natureza aromática, formada por um sistema heterogêneo e ramificado de estruturas de fenilpropano unidas por ligações éter e carbonocarbono, a lignina Kraft é o principal componente orgânico do licor negro (ZHU et al., 2015), subproduto da polpação Kraft da indústria de celulose (SOSA, 2007). Segundo os mesmos autores, este licor negro é majoritariamente utilizado como fonte de energia, através de sua queima nas caldeiras de recuperação química. $\mathrm{O}$ aumento da produção de celulose nos últimos anos (IBÁ, 2020), trouxe, por consequência, o aumento da geração deste subproduto, e as fábricas não têm conseguido realizar toda sua conversão em energia, devido à capacidade limitada das caldeiras de recuperação (SANTOS, 2016). Inúmeras pesquisas estão sendo desenvolvidas com intuito de encontrar melhor aproveitamento da lignina Kraft contida neste licor (GOUVEIA et al., 2018).

Uma alternativas é a inclusão da lignina Kraft na indústria de adesivos para madeira, pois, por ser de origem renovável e possuir estrutura química polifenólica, ela apresenta grande potencial na

\footnotetext{
${ }^{1}$ Universidade Federal de Viçosa

* Email: lari.carvalhoufv@gmail.com
} 
substituição do fenol na síntese de adesivos fenólicos para madeira (ALONSO et al., 2004).

$\mathrm{O}$ eucalipto é o gênero florestal com maior área plantada no Brasil, e é a principal matéria-prima da polpação celulósica de fibras curtas (IBÁ, 2020). A lignina de eucalipto é formada a partir da polimerização desidrogenativa dos alcóois sinapílico e coniferílico que, depois de incorporados na estrutura da lignina, recebem as denominações siringil (lignina $\mathrm{S}$ ) e guaiacil (lignina $\mathrm{G}$ ), esta última presente em menor quantidade (COLODETTE et al., 2015)A estrutura do tipo siringil apresenta menor reatividade que a guaiacil, devido à presença de grupos metil ligados ao carbono 3 e 5 das unidades de fenilpropano, diminuindo os sítios ativos para reação com o formaldeído (ABDELWAHAB; NASAR, 2011).

Apesar da baixa reatividade, a lignina Kraft ainda é considerada o substituto mais promissor dos compostos fenólicos não renováveis, devido à sua disponibilidade imediata e baixo custo (HU et al., 2011).

Neste trabalho objetivou-se sintetizar adesivos lignina-fenol-formaldeído, caracterizar suas propriedades e avaliar a resistência ao cisalhamento na linha de cola. A partir destes resultados, analisar a viabilidade do uso da lignina Kraft de eucalipto como substituta ao fenol em adesivos para madeira.

\section{MATERIAL E MÉTODOS}

Para síntese dos adesivos, utilizou-se lignina Kraft de eucalipto, em pó, precipitada do licor negro por acidifcação, com as seguintes propriedades: $\mathrm{pH} 4,91$; lignina solúvel $8,43 \%$, lignina insolúvel $88,44 \%$, e lignina total $96,86 \%$; relação S/G 2,21 e teor de cinzas $1,63 \%$. Também foram utilizados os reagentes fenol P.A. - A.C.S. (massa molecular $94,11 \mathrm{~g} / \mathrm{mol}$ ), formaldeído solução P.A. 37 \% (massa molecular 30,03 g/mol), hidróxido de sódio em pérolas P.A. ACS (massa molar $40 \mathrm{~g} / \mathrm{mol}$ ) e metanol (álcool metílico) P.A. - ACS $100 \%$ (massa molar $32,04 \mathrm{~g} / \mathrm{mol}$ ).

Além disso, foram utilizadas lâminas de madeira de Pinus sp., de resistência ao cisalhamento (média) de 99,08 Kgf $/ \mathrm{cm}^{3}$, para confecção das juntas coladas.

O adesivo fenol-formaldeído testemunha (T1) foi sintetizado seguindo a metodologia descrita por Khan et al. (2004), adicionando formaldeído $(81,08 \mathrm{~g})$, fenol fundido $(48,45 \mathrm{~g})$ e hidróxido de sódio (solução $50 \%$ ) $(4,80 \mathrm{~g})$ em um balão de fundo chato, de duas juntas, acoplado a um condensador reto e um termômetro. $\mathrm{O}$ conjunto foi aquecido à \pm $85{ }^{\circ} \mathrm{C}$ por aproximadamente $2 \mathrm{~h}$, sob agitação, com adição de 3 cargas de hidróxido de sódio $(4,84 \mathrm{~g}) \mathrm{em}$ tempos pré-determinados. Ao fim da síntese, o adesivo foi resfriado até atingir $25^{\circ} \mathrm{C}$.
Para a síntese dos adesivos lignina-fenolformaldeído, adaptou-se a metodologia anterior, substituindo o fenol por lignina Kraft de eucalipto nas proporções 25, 50, 75 e $100 \%$ em massa (T2, T3, T4 e T5, respectivamente). Na síntese dos adesivos lignina-fenol-formaldeído adicionaram-se $20 \mathrm{~g}$ de metanol à formulação adesiva, com o objetivo de retardar as reações de hidroximetilação durante a síntese e ajustar a viscosidade do adesivo final. Os adesivos foram caracterizados quanto ao gel time, $\mathrm{pH}$, teor de sólidos e viscosidade.

Confeccionaram-se juntas coladas para realização dos testes de resistência ao cisalhamento, constituídas de duas tábuas de Pinus sp. de dimensões de $40 \times 10 \times 0,5 \mathrm{~cm}$, aclimatadas até atingirem $\pm 8 \%$ de umidade, base seca,. As juntas foram produzidas aplicando-se $250 \mathrm{~g} / \mathrm{m}^{2}$ de adesivo, em face dupla, sobrepostas, pré-prensadas por $6 \mathrm{~min}$ e então prensadas a $180{ }^{\circ} \mathrm{C}$, durante $8 \mathrm{~min}$ e $1,18 \mathrm{Mpa}$ de pressão, em prensa hidráulica. Foram seccionados 60 corpos-de-prova para cada tratamento, conforme a ASTM D-2339 2008 (ASTM, 2011), que foram submetidos ao teste de resistência ao cisalhamento na linha de cola, nas condições seca - após a aclimatação a $20{ }^{\circ} \mathrm{C}$ e $65 \%$ de UR - e úmida - após 24 horas de imersão em água deionizada. Os dados foram submetidos à análise de variância e, e quando significativos, foi aplicado o teste de Scott Knott $(\mathrm{P}<0.05)$.

\section{RESULTADOS E DISCUSSÕES}

Na Tabela 1 são apresentados os resultados da caracterização dos adesivos sintetizados.

TABELA 1. Valores médios das propriedades dos adesivos sintetizados.

\begin{tabular}{ccccc}
\hline Tratamento & $\begin{array}{c}\text { Gel time } \\
(\mathrm{s})\end{array}$ & $\mathrm{pH}$ & $\begin{array}{c}\text { Teor de } \\
\text { sólidos } \\
(\%)\end{array}$ & $\begin{array}{c}\text { Viscosidade } \\
(\mathrm{cP})\end{array}$ \\
\hline $\mathrm{T} 1$ & $190 \mathrm{~A}$ & $11,58 \mathrm{~A}$ & $47 \mathrm{~A}$ & $1033 \mathrm{~B}$ \\
$\mathrm{~T} 2$ & $125 \mathrm{~B}$ & $11,49 \mathrm{~A}$ & $41 \mathrm{C}$ & $47 \mathrm{E}$ \\
$\mathrm{T} 3$ & $104 \mathrm{C}$ & $11,15 \mathrm{~B}$ & $43 \mathrm{~B}$ & $567 \mathrm{D}$ \\
$\mathrm{T} 4$ & $82 \mathrm{D}$ & $11,12 \mathrm{~B}$ & $47 \mathrm{~A}$ & $880 \mathrm{C}$ \\
$\mathrm{T} 5$ & $104 \mathrm{C}$ & $10,92 \mathrm{~B}$ & $35 \mathrm{D}$ & $1600 \mathrm{~A}$
\end{tabular}

Onde: T1: adesivo testemunha; e T2, T3, T4 e T5, correspondem às substituições de $(25,50,75$ e $100 \%$ de lignina). Médias seguidas da mesma letra, na coluna, não diferem entre si pelo teste de Scott Knott a $5 \%$ de significância.

Observaram-se diferenças significativas entre todos os resultados de gel time, com exceção de T3 e T5; o maior valor foi obtido no adesivo testemunha, indicando que a adição de lignina Kraft de eucalipto reduziu o tempo de polimerização; não foi possível estabelecer relação entre a quantidade de lignina adicionada e o gel time. 
Com relação ao $\mathrm{pH}$, o maior valor foi obtido para T1 $(11,58)$, estatisticamente igual a T2; T3, T4 e T5 foram estatisticamente iguais para esta propriedade; porém, observou-se queda nos valores à medida que a lignina Kraft foi adicionada aos adesivos, o que era esperado, devido ao caráter ácido da lignina Kraft $(4,91)$.

O maior valor de teor de sólidos foi observado em T1 e T4 (47 \%); T2, T3 e T5 foram estatisticamente diferentes. O TS de T2, T3 e T4 aumentou diretamente em relação à quantidade de lignina, porém em T5 houve uma queda brusca no valor desta propriedade. Uma hipótese para este comportamento é a de que, na ausência de fenol, a lignina e o formaldeído não reagiram, não havendo ligação química que aprisione o formaldeído; dessa forma, todo o formaldeído contido em T5 volatilizou na análise de TS.

Pode-se estabelecer relação direta entre a quantidade de lignina Kraft e viscosidade dos adesivos lignina-fenol-formaldeído, pois observou-se aumento no valor desta propriedade conforme incrementou-se a quantidade de lignina, além de diferenças estatísticas entre todos os tratamentos.

Na Figura 1 apresenta-se os resultados do teste de resistência ao cisalhamento na linha de cola dos adesivos, nas condições seca e úmida.

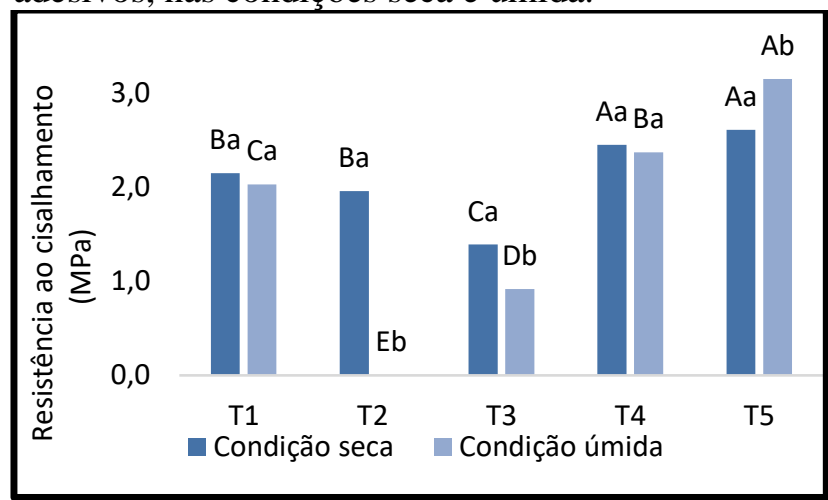

FIGURA 1. Valores médios da resistência ao cisalhamento na linha de cola, em condição seca e úmida (Mpa). Onde: T1: adesivo testemunha; e T2, T3, T4 e T5, correspondem às substituições de $(25,50$, 75 e $100 \%$ de lignina). Médias seguidas da mesma letra, na coluna, não diferem entre si pelo teste de Scott Knott a 5\% de significância.

Comparando os tratamentos entre si, considerando a condição de ensaio "seca" (letras maiúsculas sobre as colunas escuras), os maiores valores de resistência foram observados em T5 e T4, estatisticamente iguais entre si e superiores à T1; T1 e T2 foram iguais estatisticamente, e T3 apresentou o menor valor.

Já no ensaio em condição úmida (letras maiúsculas sobre as colunas claras), todos os tratamentos foram estatisticamente diferentes entre si. T5 apresentou maior resistência ao cisalhamento, seguido de T4, e só então, da testemunha T1. Em T2 nenhum corpo-de-prova resistiu ao ensaio.

Analisando os resultados do ensaio, por tratamento, nas condições seca e úmida (letras minúsculas sobre as colunas de cada tratamento), em T5 observou-se que a resistência na condição úmida foi superior à condição seca.

\section{CONCLUSÃO}

Concluiu-se que os adesivos T4 e T5 possuem maior potencial para aplicação industrial, visto que os valores obtidos no teste de resistência ao cisalhamento foram superiores aos demais tratamentos, incluindo a testemunha. Ainda que os valores obtidos nas demais propriedades dos adesivos não estejam exatamente dentro dos parâmetros ótimos, são passíveis de correção, bastando pequenos ajustes nas condições de reação, a exemplo da viscosidade, que pode ser ajustada com adição de cargas.

\section{REFERÊNCIAS}

ABDELWAHAB, N. A., NASSAR, M. A. Preparation, optimisation and characterisation of lignin phenol formaldehyde resin as wood adhesive. Pigment \& Resin Technology, London, v. 40, n. 3, p. 169-174, 2011.

ALONSO, M. V., OLIET, M., PEREZ, J. M., ECHEVERRIA, J. Determination of curing kinetic parameters of lignin-phenol-formaldeyde resol resins by several dynamic differential scanning calorimetry methods. Thermochimica Acta, Amsterdam, v. 419, n. 4, p. 535-538, Aug. 2004. Disponível em <<Goto ISI>://WOS:000223762400021>.

AMERICAN SOCIETY FOR TESTING AND MATERIALS - ASTM. Annual book of ASTM standards: adhesives: D2339-98. Philadelphia, 2000. 600 p.

COLODETTE, Jorge Luiz; GOMIDE, José Lívio; CARVALHO, Danila Morais de. Composição química de materiais lignocelulósicos. In: COLODETTE, Jorge Luiz; GOMES, Fernando José Borges. Branqueamento de polpa celulósica: da produção da polpa marrom ao produto acabado. Viçosa: Ufv, 2015. p. 33-58.

GOUVÊA, Adriana de Fátima Gomes et al. Lignina do Licor Negro Kraft de Eucalipto: Usos, Extração e Caracterização. In: GOUVÊA, Adriana de Fátima Gomes; CARVALHO, Ana Márcia Macedo Ladeira de; CARNEIRO, Angélica de Cássia Oliveira. Extração da lignina Kraft de eucalipto e uso para energia. Curitiba: Appris, 2018. p. 15-43

HU, Lihong et al. METHODS TO IMPROVE LIGNIN'S REACTIVITY AS A PHENOL SUBSTITUTE AND AS REPLACEMENT FOR OTHER PHENOLIC COMPOUNDS: A BRIEF REVIEW. Bioresources.com, Carolina do Norte, v. 3, n. 6, p.3515-3525, maio 2011. 
IBÁ INDÚSTRIA BRASILEIRA DE

ÁRVORES. Relatório Anual Ibá 2020. São Paulo: Café

Art, 2020. Disponível em:

https://iba.org/datafiles/publicacoes/relatorios/relatorioiba-2020.pdf. Acesso em: 10 nov. 2020.

KHAN, M. A., ASHRAF, S. M., MALHOTRA, V. P. Development and characterization of a wood adhesive using bagasse lignin. International Journal of Adhesion and Adhesives, v. 24, n. 6, p.485-493, 2004.

RINALDI, Roberto et al. Paving the Way for Lignin Valorisation: Recent Advances in Bioengineering, Biorefining and Catalysis. Angewandte Chemie International Edition, [s.1.], v. 55, n. 29, p.8164-8215, 17 jun. 2016. Wiley.

SANTOS, L. C.. Síntese e caracterização de adesivos lignina-fenol-formaldeído para madeira e derivados. Dissertação (Mestrado), UFV, Viçosa, 2016.

SOSA, I. R. H. Sistemas multiagentes para controle inteligente da caldeira de recuperação. 2007. 177 p.

Tese (Doutorado em Engenharia Química) - Escola politécnica da Universidade de São Paulo, São Paulo, 2007.

Zhu, W., HANS, T. Precipitation of lignin from softwood black liquor: an investigation of the equilibrium and molecular properties of lignina. BioResources, Carolina do Norte, v. 1, n. 10, p.1696-1714, 2015. 